\title{
BMJ Open Effectiveness of the Labour Inspection Authority's regulatory tools for work environment and employee health: study protocol for a cluster-randomised controlled trial among Norwegian home-care workers
}

Anne-Marthe R Indregard, ${ }^{1}$ Stein Knardahl, ${ }^{2}$ Jan Shahid Emberland, ${ }^{2}$ Øivind Skare, ${ }^{3}$ Håkon A Johannessen (iD ${ }^{1}$

To cite: Indregard A-MR, Knardahl S, Emberland JS, et al. Effectiveness of the Labour Inspection Authority's regulatory tools for work environment and employee health: study protocol for a cluster-randomised controlled trial among Norwegian homecare workers. BMJ Open 2019:9:e031226. doi:10.1136/ bmjopen-2019-031226

- Prepublication history for this paper is available online. To view these files, please visit the journal online (http://dx.doi. org/10.1136/bmjopen-2019031226).

Received 24 April 2019 Revised 20 September 2019 Accepted 31 October 2019

Check for updates

(C) Author(s) (or their employer(s)) 2019. Re-use permitted under CC BY-NC. No commercial re-use. See rights and permissions. Published by BMJ.

For numbered affiliations see end of article.

Correspondence to Dr Håkon A Johannessen; hajo@stami.no

\begin{abstract}
Introduction There is a need to evaluate whether, and to what degree, labour inspections or other regulatory tools have the desired effects on psychosocial, organisational and mechanical work environment, and employee health. The Norwegian Labour Inspection Authority (NLIA) uses different tools and strategies to enforce compliance with occupational safety and health (OSH) legislation. The aim of the present study is to evaluate the effects of labour inspections and other regulatory tools employed by the NLIA. The home-care service is one of the fastest growing occupations and a prioritised area for the NLIA, hence the present study will investigate regulatory tools in this sector.
\end{abstract}

Methods and analysis The research project has been designed as a longitudinal, cluster-randomised, controlled trial and will be conducted among Norwegian homecare workers. The objective of the research project is to evaluate the effects of the NLIA's regulatory tools (inspection and guidance) on: (1) compliance with $\mathrm{OSH}$ legislation and regulation; (2) psychosocial, organisational and mechanical work environment; (3) employee health in terms of musculoskeletal and mental health complaints; and (4) sickness absence. Public home-care services have been randomised to three intervention groups and one control group. Home-care services in the intervention groups will receive one of three intervention activities from the NLIA: (1) inspection from the Labour Inspection Authority; (2) guidance through an online interactive risk-assessment tool; and (3) guidance on psychosocial, organisational and mechanical work environment through workshops. The interventions will be performed at the organisational level (home-care service), and the effects of the interventions on the working environment and health complaints will be measured at the individual level (homecare employees).

Ethics and dissemination This project has been approved by the Regional Committees for Medical and Health Research Ethics (REC) in Norway (REC South East) (2018/2003/REK sør-øst C), the Norwegian Center for Research Data (566128), and will be conducted in
Strengths and limitations of this study

- Large-scale cluster-randomised controlled trial with an extended follow-up which may yield findings that are generalisable to real world-settings.

- National registry data on sickness absence will offer objective data on the effect of work place interventions on sickness absence rates.

- Adequate statistical power to detect small effects on measures of work environment and employee health associated with the work place interventions.

- Reliance on online and self-report assessments is likely to lead to higher levels of sample attrition and/ or incomplete and missing data.

accordance with the World Medical Association Declaration of Helsinki. The results will be reported in international peer-reviewed journals.

Trial registration number NCT03855163.

\section{INTRODUCTION}

The burden of occupational injuries and work-related diseases remain unacceptably high worldwide. A recent study estimated that 2 million deaths annually can be attributed to work-related diseases and 0.3 million deaths annually to occupational injuries. ${ }^{1}$ Both the International Labour Office and the World Health Organization view enforcement of occupational safety and health (OSH) legislation and regulation as essential to maintain employee safety and health. ${ }^{23}$ Recent reviews suggest that labour inspections improve compliance with $\mathrm{OSH}$ requirements and may reduce the incidence of occupational injuries. ${ }^{4-6}$ Nevertheless, two other reviews underlined the need for conducting experimental studies to produce decisive proof. ${ }^{78}$ 
Moreover, there is a major research gap in the effect of labour inspections on psychosocial work environment and the ability of the inspections to prevent work-related musculoskeletal and mental health complaints. ${ }^{4}$ The overarching aim of the present study is to evaluate effectiveness of the Norwegian Labour Inspection Authority's (NLIA) regulatory tools for psychosocial, organisational and mechanical work environment, and employee health.

Non-fatal health losses caused by musculoskeletal and mental disorders constitute a large and increasing proportion of the disease burden in the Norwegian population. ${ }^{9}$ It is well documented that mechanical and psychosocial exposures at work can affect employee health and increase the risk of sickness absence and disability retirement. ${ }^{10-13}$ Recent studies of the Norwegian working population have suggested that $40 \%$ of cases involving lower back pain $^{14}$ and $25 \%$ of those involving mental distress ${ }^{15}$ can be attributed to psychosocial or mechanical work factors.

The health and social sector in general, and the homecare sector in particular, show high levels of sickness absence and disability retirement related to musculoskeletal pain and mental distress, ${ }^{16}{ }^{17}$ and home-care organisations continue to experience challenges recruiting and retaining employees. ${ }^{18}$ Due to reconstruction of healthcare systems in many industrialised nations, there has been a shift from healthcare services provided in institutions to home based caring. ${ }^{19}$ In addition to a reconstruction of the healthcare system, a demographic shift due to an increasing proportion of elderly people, has led to a tremendous growth in the home care sector during the past decade, ${ }^{20}$ and the growth is assumed to continue. Although the home-care service is one of the fastest growing occupations, there are limited research describing its' OSH experiences to provide an evidence base for a safe work environment that protect employee health.

The NLIA is a government agency under the authority of the Ministry of Labour and Social Affairs. The overarching goal for the NLIA is to ensure a fully adequate working environment, safe employment conditions and meaningful work for all employees. The NLIA is responsible for ensuring that enterprises comply with the requirements in the $\mathrm{OSH}$ legislation and regulation. In addition, the NLIA provides information and advice to employers and workers concerning the most effective means of complying with the legal requirements. Research on labour inspections has mainly been limited to construction and manufacturing industries, which provide a limited base for generalisation to other sectors, such as the health and social sector. ${ }^{5}$ There is an urgent need for well-designed evaluations, such as randomised controlled trials, to be able to draw valid conclusions. ${ }^{8}$

\section{Objectives and research questions}

To respond to this research gap, the primary objective of the current research project is to evaluate the effects of the Labour Inspection Authority's regulatory tools (inspection and guidance) on the psychosocial, organisational and mechanical work environment and employee health. To fulfil this objective, the following research questions will be addressed by a longitudinal, cluster-randomised, controlled trial:

1. To what degree can the Labour Inspection Authority's regulatory tools (inspection, guidance through workshop or online risk-assessment tool) lead to compliance with OSH legislation and regulation?

2. To what degree can the Labour Inspection Authority's regulatory tools influence

a. Psychosocial work factors?

b. Organisational work factors?

c. Mechanical work factors?

3. To what degree can the Labour Inspection Authority's regulatory tools reduce

a. Musculoskeletal complaints among employees?

b. Mental health complaints among employees?

4. To what degree can the Labour Inspection Authority's regulatory tools reduce sickness absence?

Figure 1 illustrates the process through which $\mathrm{OSH}$ regulations are thought to provide the basis of a safe and healthy work environment and an important basis for primary prevention in terms of employee health and wellbeing. As shown in the figure, we will evaluate whether, and to what degree, the regulatory tools lead to compliance with $\mathrm{OSH}$ legislation and regulation (research

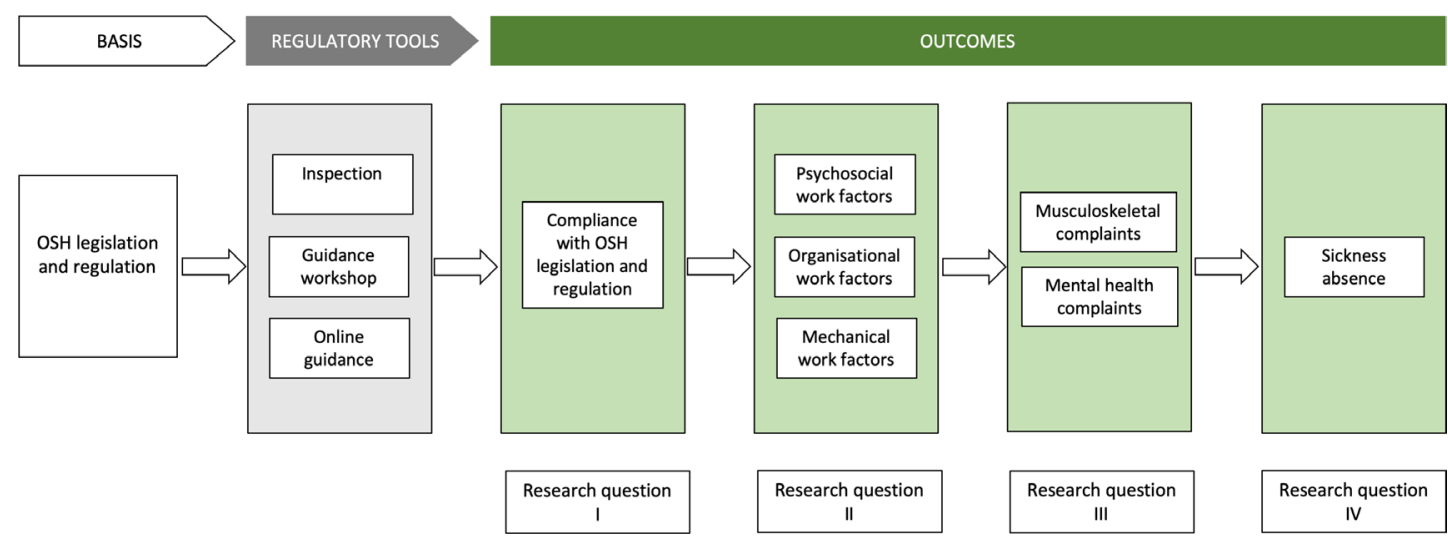

Figure 1 Illustration of the process from OSH legislation and regulation to the different outcomes. OSH, occupational safety and health. 
question 1). We will investigate if this level of compliance with OSH legislation and regulation will have effects on psychosocial, organisational and mechanical work factors (research question 2) and an effect on employee health (research question 3). The last part of this study will be to evaluate whether, and to what degree, this process leads to increased work ability and reduction of sickness absence (research question 4).

\section{METHODS AND ANALYSIS}

The protocol for the current research project has been created in accordance with the recommendations outlined in the Standard Protocol Items: Recommendations for Interventional Trials (SPIRIT) statement guidelines. $^{21}$

\section{Trial design and research setting}

The current project will be performed among public home-care workers. According to the Act Relating to Municipal Health Services ${ }^{22}$ each Norwegian municipality shall ensure that persons living in the municipality are offered the necessary health services. Therefore, each municipality has a public home-care service. For the current project, we contacted 132 municipalities in December 2018 and invited their public home-care services to participate in the project.

The current research project is designed as a longitudinal, cluster-randomised, controlled trial to evaluate the short-term and long-term effects of inspection and guidance on compliance with OSH legislation and regulation, the work environment and employee health. ${ }^{23}$ Randomisation will be conducted at the organisational level with municipalities as units. The interventions will be performed at the organisational level. Outcome measures relating to the work environment factors and health complaints will be assessed at the individual level (among home-care workers), and outcome measures to evaluate compliance with $\mathrm{OSH}$ legislation will be assessed at the group level (among home-care organisations).

Figure 2 shows a flow chart of the timeline for the three intervention groups and the control group, and shows the project's various activities starting with enrolment in autumn 2018 to the last follow-up assessment in November 2020. The trial will comprise four arms, and the eligible municipalities will be randomised to three intervention groups and one control group. The three different interventions given by the Labour Inspection Authority will be as follows: (1) Inspection by the Labour Inspection Authority; (2) guidance provided in workshops relating to the psychosocial, organisational and mechanical work environment; and (3) guidance provided as an online interactive risk-assessment tool.

Two months before the Labour Inspection Authority performs its interventions, a baseline questionnaire will be sent to all home-care workers employed in the included municipalities. This questionnaire assesses self-reported psychosocial, organisational and mechanical work factors, and employee health. The effects of the different interventions will be evaluated through questionnaires at 6,12 and 20 months after the interventions.

To evaluate how the interventions have been implemented, process evaluation will be performed immediately and 2 months after the interventions. Finally, to evaluate the effects of the interventions on compliance with $\mathrm{OSH}$ legislation and regulation, all organisations will receive an inspection from the Labour Inspection Authority 14 months after the interventions.

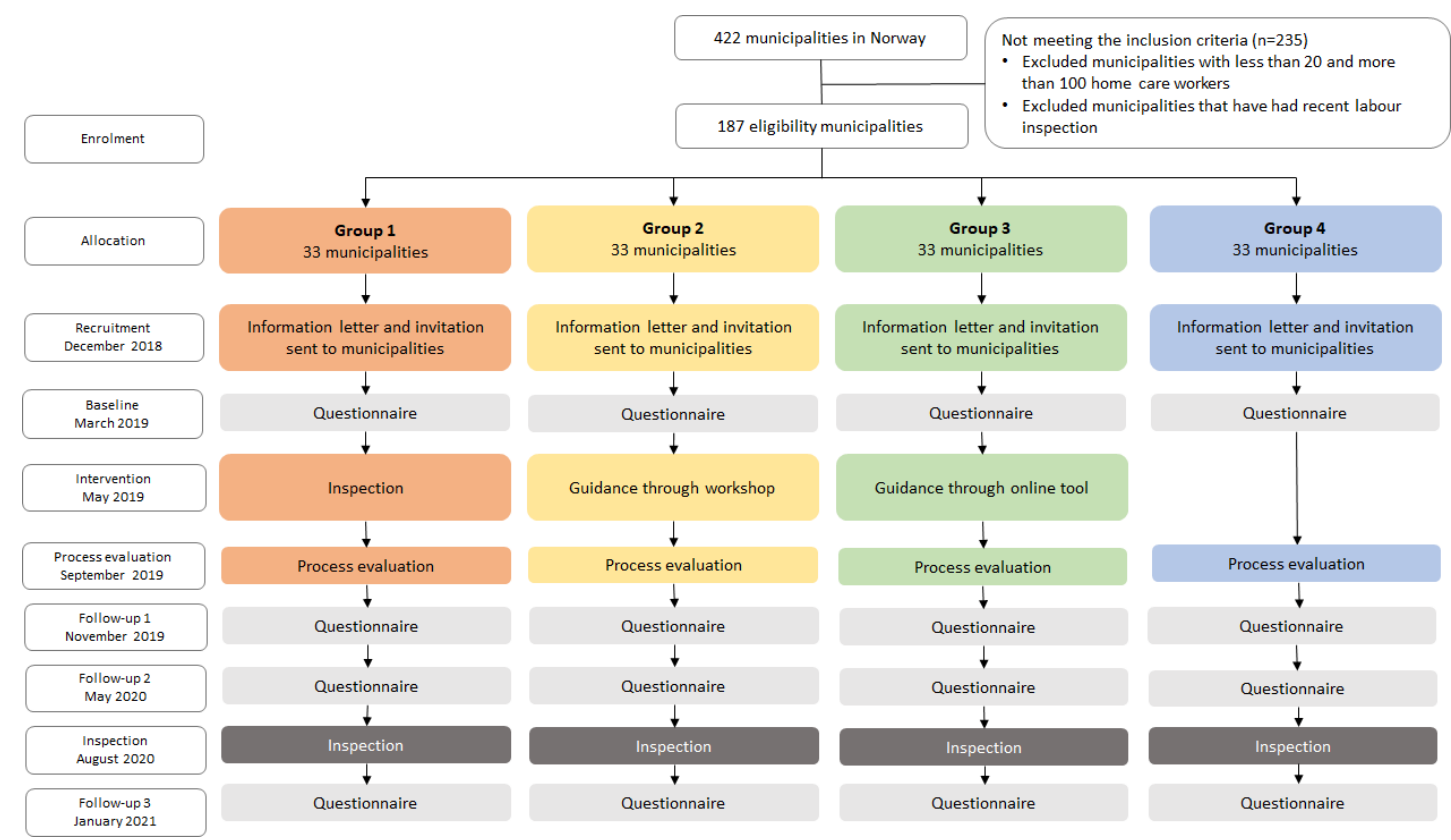

Figure 2 Flow chart illustrating the timeline of the research project. 


\section{Participants and procedure}

Home-care can be defined as 'professional care provided at home to adult people with formally assessed needs', ${ }^{24}$ and may include professional services (eg, nursing) and home support services (eg, personal care and housekeeping).

\section{Eligibility criteria for municipalities (clusters)}

Norway has 422 municipalities (1 January 2019). The number of home-care workers employed in the public home-care service in each municipality varies greatly, from three people employed in small municipalities to over 4000 employed in large municipalities. Nevertheless, in $75 \%$ of these 422 municipalities, the number of employed home-care workers are fewer than 126. To reduce intracluster variability and the sample size needed, only municipalities with 20-100 home-care workers employed in the public home-care service were assessed for eligibility. Municipalities that received an inspection from the Labour Inspection Authority in 2017 or 2018 were not eligible. After exclusion of 235 municipalities, 187 municipalities were eligible for the interventions (see figure 2).

\section{Eligibility criteria for home-care workers (individuals)}

All employees in the public home-care service in the included municipalities are eligible.

\section{Randomisation of municipalities}

Random sampling and allocation were performed using IBM SPSS Statistics version 25. Of the 187 eligible municipalities, 132 municipalities were drawn randomly and allocated to one of the interventions groups or the control group.

\section{Recruitment of home-care workers}

After the allocation of municipalities, participating municipalities will be required to complete and return a form providing an overview of their employees with contact information. Subsequently, all employees will receive an email with information about the study and an invitation to participate. The email will include a link to the webbased questionnaire. In addition, an SMS with short information about the study will be sent at the same time as the email. The SMS will contain a unique code for logging into the web-based questionnaire. The written information will explain the aims of the study and assurance that the responses will be treated confidentially and in strict accordance with the general guidelines and specific license from The Norwegian Data Protection Authority. Employees will be given the opportunity to complete the questionnaire at work, but it will also be possible for them to complete the questionnaire at home or any other location. Each respondent will have the opportunity to $\log$ into the web-based questionnaire an unlimited number of times to change or complete their answers during the survey period.

\section{Interventions}

Inspection

Labour inspection at the workplace is an essential part of the labour administration system, and a fundamental function of the Labour Inspection Authority is to enforce compliance with labour legislation. The NLIA performed nearly 14000 inspections in different sectors and organisations throughout $2017 .^{25}$ For the current project, the Labour Inspection Authority will perform inspections according to standard procedures in all public homecare services in the selected municipalities (group 1). Three weeks in advance of the inspection, a letter to announce the inspection and information about it will be sent to the participating workplaces. The inspections will target exposures related to the psychosocial, organisational and mechanical work environment. Two trained inspectors will visit each home-care unit to oversee that the enterprise complies with the requirements of The Working Environment Act. They will use a checklist that includes relevant items enshrined in the OSH legislation and regulation. In addition to the systematic observation of the work environment, the inspectors will give information and guidance to ensure compliance with labour requirements. After the inspection, the labour inspectors will prepare a report describing the current work environment and identifying the work factors that were checked, any non-compliance with legal requirements and how these should be followed up by the organisation. If necessary, the Labour Inspection Authority can enforce compliance with OSH legislation and regulation through a warning, an order to comply with the law or a citation. If necessary, it is also possible to close down the workplace.

\section{Guidance}

The Labour Inspection Authority has long experience in providing guidance on topics related to the means through which workplaces can ensure compliance with the OSH legislation and regulations. The current project will evaluate the effect of guidance through workshops and a new online interactive risk-assessment tool.

\section{Guidance through workshops}

Home-care organisations allocated to receive guidance through workshops (group 2) will receive an invitation to participate in a workshop arranged by the Labour Inspection Authority. The leader and representatives of the employees will be invited to participate. It is desirable that at least two employees from each home-care service participate at the workshop. Five to seven home-care services will be assigned to workshops based on geographical region. Before the workshop, each workplace will receive information on the topics (ie, psychosocial, organisational and mechanical work environment, and employee health) and will be asked to prepare a presentation on relevant issues arising from their own work environment. Two trained labour inspectors will participate in the workshop and give guidance based on the issues presented at that workshop. The main purposes of this method are to 
highlight relevant issues in the home-care sector and to provide guidance as to how the organisation can work systematically to reduce adverse psychosocial, organisational and mechanical work exposures. The basis for the guidance will be the OSH legislation and regulations, and the overarching aim is to motivate and ensure that the psychosocial, organisational and mechanical work environment comply with the legislation requirements.

\section{Guidance through an online interactive risk-assessment tool}

The online interactive risk-assessment tool is a wellestablished, free tool that companies can use to improve their systematic OSH work in accordance with national OSH regulations. This tool was originally developed to aid employers and employee representatives in selected industries and sectors. The home-care sector was not initially selected as a target group. A custom tool was developed for the home-care sector parallel to designing the present study. During the study period, the tool will only be available for the municipalities allocated to intervention group assigned to get guidance through this riskassessment tool.

The tool comprises a checklist with a set of questions about the organisational, mechanical and psychosocial work environment risks that are typical for the home-care sector. The questions are supplemented with guiding text explaining how and why the work environment factors may involve risk for work-related illness, injury and disease. The employer and employees answer the questions together, and the tool suggests measures to reduce the identified risks in the work environment of the company. At the end of the risk-assessment, an action plan that lists the measures, person responsible and deadline for implementation is produced.

The home-care organisations that are included in this intervention group (group 3) will each receive an email with information about the risk-assessment tool along with a link to the web page, a username and password. The main purpose of this method of guidance is to make statutory risk-assessments easier for enterprises by offering them a specific tool and to help them include relevant risk factors in the assessment by offering a check-list with predefined sector-specific risks. Although the predefined risks are based on well-known risks in the home-care sector, additional risk factors may be relevant and the companies can therefore add custom risk factors during the process. Ultimately, the tool assists home-care organisations in identifying risks present in their work environment and suggests relevant preventive measures that, in turn, may reduce the risk of work-related illness, injury and disease.

\section{Control group}

The control group will receive the usual care, meaning that no planned intervention will be implemented in the home-care services allocated to the control group (group 4). However, to evaluate any effect of the interventions on compliance with OSH legislation, all four groups will receive an inspection 14 months after the first interventions. The control group will also be asked to complete the questionnaires about the work environment and employee health at the same four times as the intervention groups.

\section{Effect evaluation}

To evaluate the effect of the Labour Inspection Authority's regulatory tools on the work environment and employee health, the study will include measures of a wide range of work factors, employee health and registry data on sickness absence. The primary and secondary outcomes are listed in table 1. These include psychosocial and mechanical working conditions, self-reported health complaints, work ability and sickness absence (a more detailed description of each measurement is given below table 1).

Data will be collected through questionnaire surveys before the interventions and at 6,12 and 20 months after the interventions. To maximise retention of participants enrolled in the study and to minimise non-response error, employees will be given the opportunity to complete the questionnaires during working hours. Participants may complete the questionnaire in Norwegian or English. The questionnaire will be available electronically through the website or on paper, and it will also be possible to complete the questionnaire in an interview. The following measurements will be repeated at each data collection point (see figure 2). The questionnaire will contain scales and questions that can be classified into the following categories.

\section{Primary outcomes}

\section{Psychosocial and organisational working conditions}

Psychosocial and organisational work factors will be assessed mainly using scales adapted from the General Nordic Questionnaire for Psychological and Social Factors at Work $\left(\mathrm{QPS}^{\text {Nordic }}\right){ }^{26}{ }^{2} \mathrm{QPS}^{\text {Nordic }}$ has been thoroughly tested for validity and reliability, and has shown good psychometric properties. ${ }^{26} 27$ The following scales from $\mathrm{QPS}^{\text {Nordic }}$ are included in the current questionnaire: quantitative demands (ie, time pressure and amount of work); decision demands (ie, demands for attention and making quick and complex decisions), learning demands (ie, demands on education and training), decision control (ie, influence on decisions regarding work tasks), control over work intensity (ie, influence on time, pace and breaks), role conflict (ie, conflicts between demands and resources, and conflicting requests), role clarity (ie, clarity of goals and objectives at work), support from the immediate superior (ie, instrumental and emotional support), empowering leadership (ie, encouragement to participate in important decisions, express different opinions and develop one's skills), fair leadership (ie, whether the immediate superior distributes work fairly and treats workers equally), predictability during the next month (ie, predictability related to tasks, co-workers and superiors), organisational support (ie, whether the social climate is encouraging/supportive and the organisation communicates its interest in employee health and well-being) and positive challenge at work (ie, usefulness of skills and knowledge, meaningfulness of work and whether work is challenging in a positive way). All scales from the QPS $^{\text {Nordic }}$ comprise 3-5 items that 
Table 1 Primary and secondary outcomes and time points for measurement

Time points for measurement after interventions

\begin{tabular}{lllll}
\hline 6 & 12 & 15 & 20 & Statistical analyses and reporting
\end{tabular}

\section{Primary outcomes}

\section{Self-reported work environment}

$\begin{aligned} & \text { Psychosocial and organisational working } \\ & \text { conditions }\end{aligned}$
Job demands, emotional demands, job control,
social support, role expectations, leadership,
bullying, violence, unwanted sexual attention

g, violence, unwanted sexual attention

Mechanical working conditions

Differences in means using linear

Squatting/kneeling, standing, upper body forward bending, awkward lifting, heavy lifting,

lifting people

\section{Self-reported health complaints}

Mental health complaints

$\checkmark 2$

mixed models/continuous outcomes

Differences in means using linear

Mental distress, exhaustion

Musculoskeletal complaints

Neck pain, back pain, headache, pain in the shoulder, upper arm, lower arm, wrist or hand, pain in hips, legs, knees or feet

Work ability

\section{Sickness absence*}

2
mixed models/continuous outcomes

Compliance with OSH legislation

Differences in means using linear mixed models/continuous outcomes

Differences in means using linear mixed models/continuous outcomes

\section{Secondary outcomes}

\section{Self-reported work environment}

New technology and work-related changes, predictability during the next month, positive challenges at work

\begin{tabular}{|c|c|c|}
\hline Self-reported health complaints & $\checkmark$ & \\
\hline sleep & & $\begin{array}{l}\text { Differences in means using linear } \\
\text { mixed models/continuous outcomes }\end{array}$ \\
\hline Occupational injuries & $\checkmark$ & $\begin{array}{l}\text { Odds ratios using mixed logistic } \\
\text { regression/dichotomous outcomes }\end{array}$ \\
\hline Systematic safety and health work & $\checkmark$ & $\begin{array}{l}\text { Odds ratios using mixed logistic } \\
\text { regression/dichotomous outcomes }\end{array}$ \\
\hline $\begin{array}{l}\text { Additional effects of labour inspection on work } \\
\text { environment and health }\end{array}$ & & $\begin{array}{l}\text { Differences in means using linear } \\
\text { mixed models/continuous outcomes }\end{array}$ \\
\hline
\end{tabular}

*Sickness absence rates will be based on doctor-certified sickness absence 1 year before and the year following the interventions.

measure the frequency of occurrence with the following response categories: $1=$ 'very seldom or never', 2='seldom', $3=$ ='sometimes', $4=$ "often' and $5=$ "very often or always'.

Emotional dissonance (ie, a discrepancy between felt and expressed emotions) will be measured by four items adapted from the Frankfurt Emotion Work Scales. ${ }^{28}$ Responses are provided on a five-point scale with the following response alternatives: 1='seldom or never',
$2=$ 'once per week', 3='once per day', $4=$ "'several times per day' and $5=$ 'several times an hour'.

Workplace bullying will be measured by first presenting a formal definition of the bullying construct and then asking the employee, 'Have you been subjected to bullying or harassment at your workplace during the past 6 months?' The response alternatives will be given on a five-point scale ranging from $1=$ "never" to $5=$ ='yes, on a daily basis'. 
Violence and threats of violence will be measured by three items developed by Statistics Norway. Employees will be asked the following questions: 'Over the past 12 months ( 6 months when follow-up), have you been the victim of violence at the workplace?' and 'Over the past 12 months (6 months when follow-up), have you been threatened at the workplace in such a way that you felt scared?' The response alternatives will be given on a fivepoint scale ranging from $1=$ 'never' to $5=$ 'yes, on a daily basis'. Employees reporting any of the 'yes' alternatives will be asked who exposed them to violence or threats of violence.

Unwanted sexual attention will be assessed using one question developed by Statistics Norway: 'Are you ever exposed to unwanted sexual attention or comments at your workplace?' The response alternatives will be given on a five-point scale ranging from $1=$ 'never' to $5=$ 'yes, on a daily basis'. Employees reporting any of the 'yes' alternatives will be asked who exposed them to unwanted sexual attention.

\section{Mechanical working conditions}

Mechanical working conditions will be measured by five items developed by Statistics Norway and used in the Norwegian survey on living conditions (work environment). ${ }^{29}$ These questions will assess the following mechanical exposures: squatting/kneeling, standing, forward bending of the upper body, awkward lifting and heavy lifting. The details have been described elsewhere. ${ }^{14}$ To account for mechanical working conditions known to be in particular relevant for home-care service employees, the following questions were adapted from Smedley and colleagues ${ }^{30}$ : 'In an average working shift, how often do you have to (1) manually transfer a client/patient between bed and chair? Or (2) manually move a client/patient around on the bed, chair or wheelchair?' (3) 'Do you perform work tasks that involve heavy physical efforts without the option of using mechanical aids?' Responses are provided on a five-point scale with response alternatives ranging from $1=$ 'never' to $5=$ 'at least 20 times'. Three other questions have been developed for the current project to measure how much time at work the employee usually spends on (1) travelling to clients, (2) office work and (3) with clients/ patients. The responses are provided on a six-point scale with response alternatives ranging from $1=$ 'never' to $6=$ 'almost all of the time'.

\section{Self-Rated health}

Self-rated health will be measured by one item pertaining to each respondent's general perception of his or her health. ${ }^{31}$ The respondents will be asked, 'How would you rate your health in general?' and the response categories are $0=$ "very bad', 1='bad', 2='moderate', 3='good' and $4=$ 'very good'.

\section{Mental health complaints}

Mental distress (symptoms of anxiety and depression) during the past week will be measured by the five-item version of the Hopkins Symptom Checklist (HSCL-10). ${ }^{32}$
Responses are provided on a four-point scale with response alternatives ranging from $1=$ 'not at all' to $4=$ ='extremely'.

Exhaustion will be measured by a six-item sub-dimension adapted from the Copenhagen Burnout Inventory. ${ }^{33}$ Responses are provided on a five-point scale ranging from $1=$ "very seldom or never' to $5=$ ="'nearly every day'.

\section{Musculoskeletal complaints}

Musculoskeletal complaints will be measured by six questions adapted from a symptom checklist ${ }^{34}$ which asks, "Have you been troubled by "neck pain", "back pain", "headache", "pain in the shoulder or upper arm", "pain in the lower arm, wrist or hand" or "pain in the hips, legs, knees or feet" during the past 4 weeks?' The response categories are 1='not troubled', 2='a little troubled', $3=$ 'intensely troubled' and $4=$ ='very intensely troubled'. Any employees who respond that they have been troubled by any complaint during the past 4 weeks will be asked whether the complaint worsens when they are at work.

To assess pain, employees will be asked to rate their general pain intensity throughout the past week using an 11-point (0-10) numeric rating scale with endpoints $0=$ 'no pain' and $10=$ "worst possible pain'. ${ }^{35}$

\section{Work ability and sickness absence}

To assess work ability the respondents will be asked to self-report their work ability at present using an 11-point $(0-10)$ numeric rating scale with endpoints $0=$ 'not able to work' and $10=$ 'work ability at its best'.

Information on sickness absence will be accessed through the Norwegian Labour and Welfare Administration. This registry provides complete registrations of all medically certified sickness absence from the first day absent, including the length and medical diagnosis. After having secured informed consent from the employees, survey data will be linked to registrations on medically certified absence through the unique 11-digit national identity number.

\section{Compliance with $\mathrm{OSH}$ legislation}

To evaluate the effects of the different interventions on compliance with OSH legislation and regulation, all organisations will be given a compliance score based on the check-list that is used for the labour inspections.

\section{Secondary outcomes}

\section{New technology and work-related changes}

Introduction and use of new technology will be assessed by three questions on implementation of new technology during the past 12 months (6 months when follow-up), involvement of employees in the implementation process and training of the employees in the use of new technology.

To assess work-related changes, the questionnaire includes three questions related to changes at the workplace during the past 3 years. The first two questions consider whether changes at work have occurred and if so, to what degree these changes have affected the work situation of the employee. The third question asks whether the 
employer has implemented a process by which all those affected by the changes can be heard.

\section{Sleep}

Sleep will be measured using the Bergen Insomnia Scale. ${ }^{36}$ This scale comprises six items that assess symptoms of insomnia. The self-rating is repeated for each item, and the respondent gives a score on an eight-point scale indicating the number of days per week when a specific symptom is experienced ( $0-7$ days, total score $0-42)$.

\section{Occupational injuries}

Information about occupational injuries will be collected by the following question, 'During the past 12 months (6 months when follow-up), have you been injured as a result of an accident at work which led to sick leave? ${ }^{29} 37$

\section{Systematic safety and health work}

Four questions will assess the degree to which the organisation works systematically to enforce the OSH regulations at work. Employees will be asked whether they have a safety representative and a working environment committee at their workplace. They will also be asked whether they have had appraisal interviews during the past year and whether they have participated in any kind of workplace interventions aiming to improve the work environment. The last part of the questionnaire will include three questions about notification of critical conditions in the workplace. Employees will be asked whether they have witnessed any critical conditions during the past 12 months $(6$ months when follow-up). Employees responding 'yes' to this question will be asked whether the condition was notified and what happened after the notification.

\section{Demographics and work characteristics}

Information on sex, age, marital status, years of education and years of employment in the current working position will be collected at the baseline. Employment status, information related to shift-work and perceived work affiliation (eg, intention to quit the job) will be collected at each data collection point.

\section{Process evaluation}

Process evaluation will be conducted to evaluate the implementation of the three interventions and the possible mechanisms by which the interventions achieve their effects. Process evaluation will combine questionnaire responses and data from employers, employees and labour inspectors. The recommendation for process evaluation of complex interventions published by the Medical Research Council guidance ${ }^{38}$ will be used as a framework for conducting and reporting the process evaluation. As illustrated in figure 2, the process evaluation will be conducted through survey questionnaires 3 months after the interventions. The questionnaires will include questions about how the intervention has been received which people in the workplace are involved in the intervention and what is being done in response to the intervention. The process evaluation will also ask to what degree the participants perceive the intervention as relevant and useful for their workplace. The organisations allocated to the control group will be asked about changes at their workplace during the same period.

\section{Statistical analyses}

Planned analysis

Analysis and reporting of the results will follow the SPIRIT guidelines for reporting randomised controlled trials. ${ }^{21}$ Baseline tables will show the mean and SD for the outcome variables and baseline characteristics for the different intervention groups (ie, gender, age, marital status, formal education, part-time employment and geographic region) Separate analyses of the longitudinal data will be performed for each primary and secondary outcome.

The statistical analyses will be divided into four parts, each corresponding to a stated research question. Because our study is a randomised trial, we do not plan to make any adjustment for baseline characteristics. However, as recommended for randomised trials, we will adjust for the baseline value for each outcome variable. The postbaseline values (6, 12 and 20 months) will be analysed using a linear mixed model for continuous outcomes, Poisson mixed models for count models and logistic mixed models for binary outcomes. Participants, work units and municipalities will be included as nested random effects. When analysing more than one post-baseline value, we will include as a fixed effect an interaction between time (follow-up 6 and 12 months) and group. This interaction represents the effect of the intervention at the two follow-up times. The primary end-point is the 12-month follow-up, and the analyses focusing on this end-point will include data obtained at 6 and 12 months.

The protocol sets up a post-test-only control group whose inclusion is intended to evaluate the compliance with $\mathrm{OSH}$ legislation and regulation, which will be measured at the 15 month inspection. ${ }^{39}$ Only post-test data will be used for group comparisons. Because of the randomisation, the expected compliance scores for the four groups will be equal if there is no intervention effect. We plan to use t tests to compare the control group with each of the three intervention groups.

We also plan to perform stratified analyses to determine whether an intervention has an effect on participants reporting the most problems at baseline. For each outcome, we will analyse the data for $25 \%$ of the participants reporting the most problems using the same analyses as described above. To control for multiple testing, we will use q values which is the false discovery rate equivalent for $p$ values. ${ }^{40}$

\section{Sample size}

Sample size calculations are based on the principles of cluster-randomised trials ${ }^{41}$ and will take into account interclass correlations of the outcome nested by the municipalities. We used the following formula to estimate the number of employees, $m$, per group ${ }^{41}$ : 


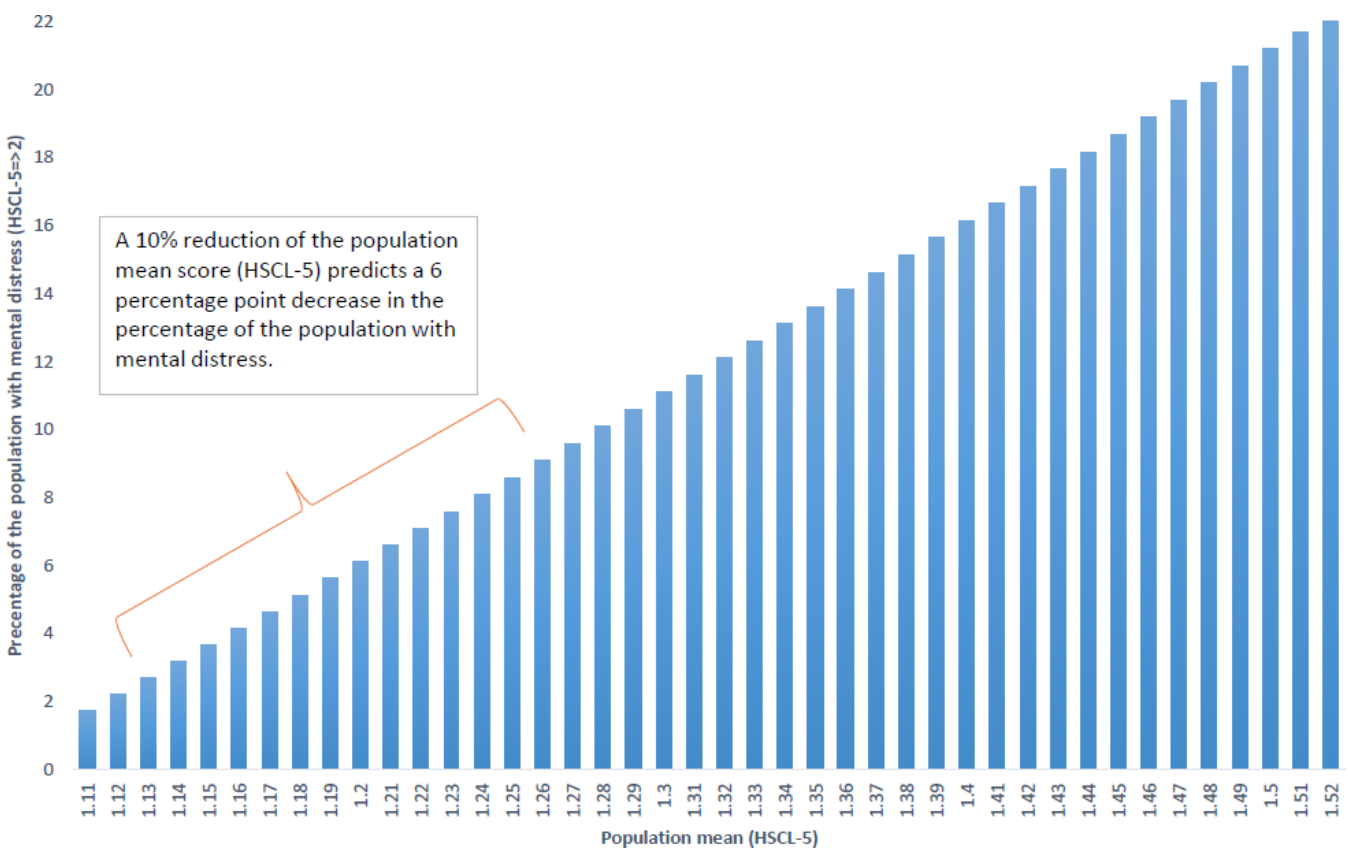

Figure 3 Percentage of the population with mental distress (HSCL-5 $\geq 2$ ) according to the different population mean scores on the HSCL-5-scale. HSCL, Hopkins Symptom Checklist.

$$
m=\frac{\left(Z_{1-\frac{\alpha}{2}}+Z_{1-\beta}\right)^{2} 2 \sigma^{2}}{\Delta^{2}}\left(1+\left\{\left(C V^{2}+1\right) \bar{n}-1\right\} \rho\right)
$$

where $Z x$ is the $x$ th percentage point of the standard normal distribution, $\Delta$ is the clinically important difference in the means of health complaints, $\sigma^{2}$ is the variance of the outcome, $\bar{n}$ is the mean cluster size (home care service employees per municipality) and CV is the corresponding coefficient of variation, and $\rho$ is the intracluster correlation coefficient (ICC).

Based on the level of 'Living-Working Conditions Survey' which was conducted by Statistics Norway in $2006,{ }^{28}$ we estimate the population mean scores for subjective musculoskeletal pain to be $1.53(\mathrm{SD}=0.58)$, and for mental distress (HSCL-5) to be 1.26 ( $\mathrm{SD}=0.45)$, among social and healthcare workers. Figures from Statistics Norway showed that each eligible municipality would recruit on average 52 employees $(\mathrm{SD}=21.9)$. The worksite level ICCs for a variety of outcomes have been estimated with a mean of 0.0163 and values ranging from 0 to $0.0650 .{ }^{42}$

An individually randomised study would require 222 employees per group. To account for clustering effects from randomised municipalities, with a conservative ICC of 0.05 , the estimate was inflated to 891 employees, with 17 municipalities recruiting 52 employees each per group. Finally, to allow for a response rate of $70 \%$ and loss to follow-up of $20 \%$, we plan to recruit 1591 employees, with 31 municipalities recruiting 52 employees in each per group.

Figure 3 illustrates the clinical implication of a $10 \%$ reduction in the population mean score of the HSCL-5 scale. To obtain these estimates, we performed a resampling (bootstrapping) of subsamples from the Norwegian Life course survey. ${ }^{29}$ This figure shows the percentage of people with an HSCL-score $\geq 2$ according to given mean HSCL population scores. Figure 3 shows that a population mean HSCL-score of 1.26 predicts that $9 \%$ of the population will have an HSCL-score $\geq 2$. Moreover, a $10 \%$ reduction of this population mean score predicts a 6 percentage point decrease in the percentage of the population with mental distress.

\section{DISCUSSION}

To our knowledge, the current study will be one of the first, cluster randomised controlled trials to assess whether the Labour Inspection Authority's regulatory tools have an effect on psychosocial work environment and employee health. The study has a sufficient sample size to detect small effects on work environment and employee health, and the work place interventions and outcomes are assessed using validated instruments. Nevertheless, there are also some limitations that should be addressed.

Intervention studies using simultaneous comparison populations may face problems related to contamination between intervention groups and the control group. ${ }^{43}$ This study has municipalities as the 'unit of randomisation' and a certain degree of 'contamination' between the municipalities are unavoidable, that is, employees in the control group may receive information regarding ongoing or planned inspection in neighbouring municipalities and consequently take action to improve their own work environment. In addition, the work places will become familiar with the study before implementation of interventions through information letters and baseline questionnaires. Hence, to control for contamination between groups, the process evaluation will be used as an information source to detect possible prevention activities executed before the NLIA implement their planned interventions. 
When designing studies and performing interventions within social science a common goal is to draw conclusion about the effects of specific interventions on some chosen outcome variables. However, the context studied often proposes complex relationships among variables and third variables may affect how and when OSH regulation have an effect on work environment and employee health. The current study includes different municipalities with their own organisation and policies that may affect how and under which conditions regulatory tools lead to compliance with OSH regulation. Nevertheless, the randomisation will balance both observed and unobserved characteristics between the groups of municipalities and thereby minimise the risk of confounding factors influencing the results. ${ }^{44}$

The appropriate length of time lags between study waves is a crucial issue in longitudinal research methodology. However, a general rule regarding the appropriate length of time interval for a study do not exist. ${ }^{45}$ We expect that an improvement in the work environment (ie, a reduction in a chronic work stressor) should have positive effect on employee health. To provide essential information about excepted effects over time, several measurement occasions with short time-lags are recommended. ${ }^{46}$ Thus, the current study measures work environment and employee health before the interventions and at 6,12 and 20 months after the interventions.

As the current study use survey questionnaires to assess working conditions and employee health, potential problems arising from self-report instruments, such as response-set tendencies and recall bias have to be discussed. To reduce measurement error the questionnaire includes validated instruments with acceptable psychometric properties. In addition, the QPSNordic instrument used in the current study, do not address issues that are inherently positive or negative, nor degrees of satisfaction and agreement. Instead the respondents are asked how often a situation occurs. ${ }^{26}$ Thus, the measurement should be insensitive to respondents' emotions and personality. Furthermore, when we use a combination of questionnaire survey and objective registry data on sickness absence, the results are less subjected to common-method bias/common method variance. ${ }^{47}$

In summary, the current study will respond to a research gap regarding the effects of the NLIA's regulatory tools on psychosocial work environment and employee health. Despite the potential limitation listed above, the findings from the study may provide crucial information regarding different regularly tools (inspection and guidance) which may have practical implications for NLIA in their work to enforce compliance with OSH legislation.

\section{ETHICS AND DISSEMINATION}

All participants are required to provide informed consent before they begin the questionnaire. The information letter explains that participation is voluntary and that participants may withdraw from the study at any time without any consequences. Self-reported data are recorded in electronic files, and no identifying information is stored with the self-reported data. The study results will be presented at national and international meetings, and in peer-reviewed publications.

\section{PATIENT AND PUBLIC INVOLVEMENT}

We have ensured research involvement and engagement by establishing a research reference group. The reference group consists of representatives from the authorities and the social partners. This reference group has contributed to making the study design relevant, participant friendly and ethically sound, and the representatives will be involved in the dissemination of study results

\section{COLLABORATION}

The research project is a collaboration between the NLIA and the National Institute of Occupational Health (STAMI). The Labour Inspection Authority will perform their inspections and guidance in the randomised municipalities. STAMI is responsible for the process evaluation and for collecting data from home-care workers about their work environment and health.

\section{Author affiliations}

${ }^{1}$ Department of Occupational Health Surveillance, National Institute of Occupational Health, Oslo, Norway

${ }^{2}$ Department of Work Psychology and Physiology, National Institute of Occupational Health, Oslo, Norway

${ }^{3}$ Department of Occupational Medicine and Epidemiology, National Institute of Occupational Health, Oslo, Norway

Acknowledgements The current project was conceived in collaboration with the Norwegian Labour Inspection Authority. The authors thank Gunn Robstad Andersen, Marianne Elvsaas Nordtømme and Yogindra Samant for valuable comments on drafts of this protocol, and Shahrooz Elka for assistance in the survey administration.

Collaborators The research project is a collaboration between the Labour Inspection Authority and the National Institute of Occupational Health (STAMI). The Labour Inspection Authority will perform their inspections and guidance in the randomised municipalities. STAMI is responsible for the process evaluation and for collecting data from home-care workers about their work environment and health.

Contributors HAJ and SK: first version of the study design. AMI: draft of the protocol manuscript. AMI, HAJ, SK, ØS and JSE: participation in the final design of the study and coordination. All authors read, revised and approved the final protocol manuscript.

Funding The project is funded by the National Institute of Occupational Health and the Norwegian Labour Inspection Authority.

Competing interests None declared.

Patient consent for publication Not required.

Ethics approval This project has been approved by the Regional Committees for Medical and Health Research Ethics (REC) in Norway (REC South East) (2018/2003/ REK sør-øst C), the Norwegian Center for Research Data (566128), and will be conducted in accordance with the World Medical Association Declaration of Helsinki.

Provenance and peer review Not commissioned; externally peer reviewed.

Data availability statement Data will be available 3 years after study completion. Data access request will be reviewed by NSD - Norwegian Centre for Research Data. URL: https://nsd.no/nsd/english/index.html. 
Open access This is an open access article distributed in accordance with the Creative Commons Attribution Non Commercial (CC BY-NC 4.0) license, which permits others to distribute, remix, adapt, build upon this work non-commercially, and license their derivative works on different terms, provided the original work is properly cited, appropriate credit is given, any changes made indicated, and the use is non-commercial. See: http://creativecommons.org/licenses/by-nc/4.0/.

\section{ORCID iD}

Håkon A Johannessen http://orcid.org/0000-0001-9974-4824

\section{REFERENCES}

1 Takala J, Hämäläinen Päivi, Saarela KL, et al. Global estimates of the burden of injury and illness at work in 2012. J Occup Environ Hyg 2014;11:326-37.

2 International Labour Office. Global strategy on occupational safety and health: conclusions adopted by the International labour conference at its 91st session, 2003, 2004.

3 World Health Organization (WHO). Declaration on workers health. Approved at the seventh meeting of the who collaborating centres for occupational health. Italy: WHO, 2006.

4 Andersen JH, Malmros P, Ebbehoej NE, et al. Systematic literature review on the effects of occupational safety and health $(\mathrm{OSH})$ interventions at the workplace. Scand J Work Environ Health 2019;45:103-13

5 Johannessen HA, Løchting I, Bakke B, et al. Effekter av myndighetstiltak på arbeidsmiljø og helse. En systematisk kunnskapsoppsummering STAMI; 2017.

6 Tompa E, Kalcevich C, Foley M, et al. A systematic literature review of the effectiveness of occupational health and safety regulatory enforcement. Am J Ind Med 2016;59:919-33.

7 Blanc F. From chasing violations to managing risks: origins, challenges and evolutions in regulatory inspections. Edward Elgar Pub, 2018.

8 Mischke C, Verbeek JH, Job J, et al. Occupational safety and health enforcement tools for preventing occupational diseases and injuries. Cochrane Database Syst Rev 2013;20.

9 Tollånes M, Knudsen A, Vollset S. Disease burden in Norway in 2016. Tidsskrift for den Norske laegeforening: tidsskrift for praktisk medicin NY raekke. 138, 2018.

10 Duijts SFA, Kant I, Swaen GMH, et al. A meta-analysis of observational studies identifies predictors of sickness absence. $J$ Clin Epidemiol 2007;60:1105-15.

11 Knardahl S, Johannessen HA, Sterud T, et al. The contribution from psychological, social, and organizational work factors to risk of disability retirement: a systematic review with meta-analyses. BMC Public Health 2017;17:176.

12 Knardahl S, Sterud T, Nielsen MB, et al. Arbeidsplassen og sykefravær - Arbeidsforhold av betydning for sykefravær.. Tidsskrift for velferdsforskning. 2016;19:179-99.

13 Stansfeld S, Candy B. Psychosocial work environment and mental health - a meta-analytic review. Scand J Work Environ Health 2006;32:443-62.

14 Sterud T, Tynes T. Work-Related psychosocial and mechanical risk factors for low back pain: a 3-year follow-up study of the general working population in Norway. Occup Environ Med 2013;70:296-302.

15 Johannessen HA, Tynes T, Sterud T. Effects of occupational role conflict and emotional demands on subsequent psychological distress: a 3-year follow-up study of the general working population in Norway. J Occup Environ Med 2013;55:605-13.

16 Dellve L, Lagerström M, Hagberg M. Work-system risk factors for permanent work disability among home-care workers: a case-control study. Int Arch Occup Environ Health 2003;76:216-24.

17 Utaaker E, Madshus AC, Flesjø M, et al. På Rett vei I hjemmetjenesten? Rapport for perioden 2002-2004, 2006.

18 Jang $Y$, Lee AA, Zadrozny M, et al. Determinants of job satisfaction and turnover intent in home health workers: the role of job demands and resources. J App/ Gerontol 2017;36:56-70.

19 Denton MA, Zeytinoğlu IU, Davies S. Working in clients' homes: the impact on the mental health and well-being of visiting home care workers. Home Health Care Serv Q 2002;21:1-27.

20 Quinn MM, Markkanen PK, Galligan CJ, et al. Occupational health of home care aides: results of the safe home care survey. Occup Environ Med 2016;73:237-45.

21 Chan A-W, Tetzlaff JM, Altman DG, et al. Spirit 2013 statement: defining standard protocol items for clinical trials. Ann Intern Med 2013;158:200-7.
22 Health and Care Services Act. Act relating to municipal health and care services, etc. (health and care services act), 2011. Available: https://app. uio.no/ub/ujur/oversatte-lover/data/lov-20110624-030-eng.pdf

23 Turner EL, Li F, Gallis JA, et al. Review of recent methodological developments in group-randomized trials: part 1-design. Am J Public Health 2017;107:907-15.

24 Genet N, Boerma WGW, Kringos DS, et al. Home care in Europe: a systematic literature review. BMC Health Serv Res 2011;11:207.

25 The Norwegian Labour Inspectorate. Årsrapport 2017 (Norwegian), 2018. Available: https://www.regjeringen.no/contentassets/052f7f772897495c b32afc2980b6898d/arsrapport-arbeidstilsynet-2017-rev.pdf

26 Dallner M, Elo A-L, Gamberale F, et al. Validation of the General Nordic Questionnaire (QPSNordic) for psychological and social factors at work. Copenhagen: Nordic Council of Ministers, 2000.

27 Wannstrom I, Peterson U, Asberg M, et al. Psychometric properties of scales in the General Nordic Questionnaire for Psychological and Social Factors at Work (QPS ${ }_{\text {Nordic }}$ ): Confirmatory factor analysis and prediction of certified long-term sickness absence. Scand J Psychol 2009;50:231-44.

28 Zapf D, Vogt C, Seifert C, et al. Emotion work as a source of stress: the concept and development of an instrument. European Journal of Work and Organizational Psychology 1999;8:371-400.

29 Statistics Norway. Samordnet levekårsundersøkelse 2009 - Tverrsnitt Tema: Arbeidsmiljø. [Coordinated Living Conditions Survey 2009 Cross Sectional. Focus: Work environment. Oslo: Statistics Norway, 2010.

30 Smedley J, Egger P, Cooper C, et al. Manual handling activities and risk of low back pain in nurses. Occup Environ Med 1995;52:160-3.

31 Idler EL, Benyamini Y. Self-Rated health and mortality: a review of twenty-seven community studies. J Health Soc Behav 1997;38:21-37.

32 Derogatis LR, Lipman RS, Rickels K, et al. The Hopkins Symptom Checklist (HSCL): a self-report symptom inventory. Behav Sci 1974;19:1-15.

33 Kristensen TS, Borritz M, Villadsen E, et al. The Copenhagen Burnout Inventory: a new tool for the assessment of burnout. Work \& Stress 2005;19:192-207.

34 Steingrímsdóttir Ólöf A, Vøllestad NK, Røe C, et al. Variation in reporting of pain and other subjective health complaints in a working population and limitations of single sample measurements. Pain 2004;110:130-9.

35 Downie WW, Leatham PA, Rhind VM, et al. Studies with pain rating scales. Ann Rheum Dis 1978;37:378-81.

36 Pallesen S, Bjorvatn B, Nordhus IH, et al. A new scale for measuring insomnia: the Bergen insomnia scale. Percept Mot Skills 2008;107:691-706

37 Johannessen HA, Gravseth HM, Sterud T. Psychosocial factors at work and occupational injuries: a prospective study of the general working population in Norway. Am J Ind Med 2015;58:561-7.

38 Moore GF, Audrey S, Barker M, et al. Process evaluation of complex interventions: medical Research Council guidance. BMJ 2015;350:h1258.

39 Søberg M. Labour Inspections and its impact on enterprises' compliance with safety regulations. Safety Science Monitor 2013;17:1-12.

40 Krzywinski M, Altman N. Comparing samples-part II. Nat Methods 2014;11:355-6.

41 Rutterford C, Copas A, Eldridge S. Methods for sample size determination in cluster randomized trials. Int $J$ Epidemiol 2015;44:1051-67.

42 Martinson BC, Murray DM, Jeffery RW, et al. Intraclass correlation for measures from a worksite health promotion study: estimates, correlates, and applications. Am J Health Promot 1999;13:347-57.

43 Deaton A, Cartwright N. Understanding and misunderstanding randomized controlled trials. Soc Sci Med 2018;210:2-21.

44 Hariton E, Locascio JJ. Randomised controlled trials - the gold standard for effectiveness research. BJOG: Int J Obstet Gy 2018;125

45 Ford MT, Matthews RA, Wooldridge JD, et al. How do occupationa stressor-strain effects vary with time? A review and meta-analysis of the relevance of time lags in longitudinal studies. Work \& Stress 2014;28:9-30.

46 Dormann C, Griffin MA. Optimal time lags in panel studies. Psychol Methods 2015;20:489-505.

47 Podsakoff PM, MacKenzie SB, Lee J-Y, et al. Common method biases in behavioral research: a critical review of the literature and recommended remedies. J Appl Psychol 2003;88:879-903. 